\title{
COPD and the gut-lung axis: the therapeutic potential of fibre
}

\author{
Annalicia Vaughan ${ }^{1,2}$, Zoe A. Frazer ${ }^{1,2}$, Philip M. Hansbro ${ }^{3,4}$, Ian A. Yang ${ }^{1,2}$ \\ ${ }^{1}$ UQ Thoracic Research Centre, Faculty of Medicine, The University of Queensland, Chermside, Brisbane, Australia; ${ }^{2}$ Department of Thoracic \\ Medicine, The Prince Charles Hospital, Brisbane, Australia; ${ }^{3}$ Centre for Inflammation, Centenary Institute, University of Technology Sydney, \\ Faculty of Science, Camperdown, Australia; ${ }^{4}$ Priority Research Centre for Healthy Lungs, Hunter Medical Research Institute, The University of \\ Newcastle, Callaghan, Australia \\ Contributions: (I) Conception and design: All authors; (II) Administrative support: None; (III) Provision of study materials or patients: None; (IV) \\ Collection and assembly of data: None; (V) Data analysis and interpretation: All authors; (VI) Manuscript writing: All authors; (VII) Final approval of \\ manuscript: All authors. \\ Correspondence to: Dr. Annalicia Vaughan. UQ Thoracic Research Centre, Room 2, Level 1, Clinical Sciences Building, The Prince Charles Hospital, \\ Rode Rd., Chermside, QLD, Australia. Email: a.vaughan@uq.edu.au.
}

\begin{abstract}
Current management strategies for chronic obstructive pulmonary disease (COPD) incorporate a step-wise, multidisciplinary approach to effectively manage patient symptoms and prevent disease progression. However, there has been limited advancement in therapies to address the underlying cause of COPD pathogenesis. Recent research has established the link between the lungs and the gut - the gut-lung axis -and the gut microbiome is a major component. The gut microbiome is likely perturbed in COPD, contributing to chronic inflammation. Diet is a readily modifiable factor and the diet of COPD patients is often deficient in nutrients such as fibre. The metabolism of dietary fibre by gut microbiomes produces anti-inflammatory short chain fatty acid (SCFAs), which could protect against inflammation in the lungs. By addressing the 'fibre gap' in the diet of COPD patients, this targeted dietary intervention may reduce inflammation, both systemically and in the airways, and value-add to the paradigm shift in respiratory medicine, from reactive to personalised and participatory medicine.
\end{abstract}

Keywords: Chronic obstructive pulmonary disease (COPD); microbiome; metagenomics

Submitted Sep 11, 2019. Accepted for publication Oct 17, 2019.

doi: $10.21037 /$ jtd.2019.10.40

View this article at: http://dx.doi.org/10.21037/jtd.2019.10.40

\section{Introduction}

Chronic obstructive pulmonary disease (COPD) is a chronic lung disease characterised by chronic airway inflammation, persistent respiratory symptoms and chronic airflow limitation, and is associated with exacerbations and comorbidities $(1,2)$. It is estimated that $4-5 \%$ of the global population or 328 million people have COPD (3). Global Burden of Disease studies have estimated that COPD causes the death of at least 2.9 million annually and it is predicted to be third leading cause of death worldwide by $2020(4,5)$. COPD also predisposes to lung cancer (6-8).

COPD management encompasses the treatment and stabilisation of disease symptoms, as well as secondary prevention strategies to manage lung function decline and the risk of exacerbation. This requires a stepwise, multidisciplinary approach. Current management strategies for COPD include both pharmacological and non-pharmacological strategies, which work to optimise lung function and prevent deterioration (1). Pharmacological interventions include short and long acting bronchodilators, anti-inflammatory agents (1) and antibiotics, particularly macrolides may be beneficial (9). Non-pharmacological interventions include smoking cessation, pulmonary rehabilitation and vaccination, among others (1). Current management strategies are effective at stabilizing the disease, yet there have been limited advances in targeted therapies to reverse the pathogenesis of COPD and the deterioration of lung function. A promising yet understudied area of COPD management is nutrition $(10,11)$. 


\section{Nutrition and COPD}

Nutrition is defined as the utilization of digested food materials by living organisms, which enables them to grow, maintain themselves and reproduce. It is a modifiable lifestyle factor and the cornerstone of good health and well-being. Poor nutrition is associated with chronic diseases, many of which have an inflammatory nature $(10,12-17)$. Nutrition is sourced through the diet, which is defined as the kinds of foods that a person habitually eats. The Western diet is characterised by frequent intakes of red/processed meat, saturated/trans-fat, refined grains, processed sugar, beer and spirits (18). The Western diet is often nutrient deficient (19) and has been associated with features of COPD including cough and sputum production, worsening airflow obstruction and systemic inflammation. Conversely, a more considered diet which includes the frequent intake of vegetables, fruit, cooking/dressing oil, cereals and legumes, whole-grains, rice/pasta, fish, low-fat dairy, poultry and water is considered a "Prudent diet" (20). Recent research has revealed an inverse relationship between dietary patterns consistent with a Prudent diet and the risk of COPD, and a direct association between a Western diet and increased COPD risk in both men and women (21-23).

A series of studies by Kaluza et al. has investigated the relationship between fruits, vegetable and fibre consumption in men and women (24-26). In women, long-term consumption of fruits but not vegetables was associated with lower risk of COPD. In men, there was a strong association between total fruit and vegetable consumption in current and former smokers but not never smokers. For fibre specifically, there was a strong inverse association between total fibre intake and COPD. In agreement with this, Shaheen $e t$ al., found a positive association between fruit, vegetables, oily fish and wholemeal cereals with $\mathrm{FEV}_{1}$ and FVC in COPD patients (20). For males only, there was a positive association with $\mathrm{FEV}_{1} / \mathrm{FVC}$ and these associations were stronger in smokers than non-smokers (20). These findings highlight the importance of a healthy diet in multiinterventional, multi-disciplinary programs to prevent and manage COPD.

\section{The gut-lung axis, the gut microbiome and respiratory disease}

The gut holds $99 \%$ of the commensal bacterial mass of the human microbiome, primarily involving the colonization of the small intestine (27). These bacteria produce metabolites and ligands, which directly aid in digestion, while indirectly regulating the immune system and inflammation (27). Absorption of these bacterial products into the circulation induces systemic effects on the host by the gut microbiome.

The gut-lung axis is the bi-directional crosstalk between the gut and the lungs (10). Disease in the lung affects the gut and vice versa $(5,10,28-32)$. These links are facilitated by the gut microbiome (33). The gut microbiome produces a number of metabolites through fermentation, the most widely recognised being short-chain fatty acids (SCFAs). SCFAs are potent anti-inflammatory molecules, which reduce chemotaxis and adherence in immune cells, while increasing the release of anti-inflammatory cytokines and inducing apoptosis $(34,35)$. The absorption of these molecules into the systemic circulation has anti-inflammatory effects in the host. Hence, the gut microbiome and SCFAs influence lung health, and vice versa, through systemic changes in the circulation induced by microbial metabolites.

\section{Dysbiosis of the gut microbiome in respiratory disease and implications for COPD}

One of the hallmarks of a 'healthy' gut microbiome is high bacterial diversity and richness (36). In a group of 'healthy' individuals, the abundance of bacterial species in the gut will differ depending on a number of personal factors, though a number of core bacterial phyla and species have been identified; Actinobacteria, Firmicutes, and Bacteroidetes are the most abundant phyla throughout the entire intestinal tract, with specific colonisation by Bifidobacterium, Lachnospiraceae, Streptococcus, Enterococcus, and Lactobacillus being regularly distinguished (37). In patients with chronic illnesses, there is a generalized perturbation of the microbiome toward increased levels of harmful bacteria, such as Clostridium and Escherichia, with reduced levels of the normal commensal bacteria (35). The mechanisms behind these changes are not well studied, though the shift in microbial profile with chronic lung diseases has been well established.

\section{Gut microbiome in asthma}

Recently, the metagenomic analysis of stool samples from 36 patients with asthma identified that the gut microbiome of the cohort had a general decrease in diversity, compared to a healthy cohort (38). Additional findings suggested that butyrate-producing bacteria, such as Faecalibacterium 
prausnitzii and Coprococcus eutactus, were depleted with direct correlation to increases in the abundance of Clostridium spp. and Eggerthella lenta (38). Functionally, the gut microbiome of the asthma patients shifted away from SCFA production and metabolism toward lipid, carbohydrate, and amino acid metabolism (38). Since asthma is characterized by abnormal Th2 cell responses, the altered SCFA production by the gut microbiome may contribute to pathogenesis through reduced anti-inflammatory butyrate activity on these cells $(38,39)$. Furthermore, it was found that there are more active virulence factors in the gut microbiome of asthma patients perpetuating their overall inflammatory state. Despite these findings, it is unknown why such changes occur in this population or if the mechanisms involved promote the pathology of the disease or vice versa.

\section{Gut microbiome in CF}

Cystic fibrosis (CF) is another chronic lung disease where alterations in the gut microbiome are observed. Compared to patients with asthma, CF patients often have chronic intestinal inflammation and are frequently prescribed regular antibiotic treatments from a young age (40). Both these factors can rapidly and potently disturb the gut microbiome. However, the importance of the gut-lung axis is also demonstrated in this disease with microbial richness being related to pathogenic lung colonisation. The metagenomic analysis of stool from 31 children with CF found that gut microbiome was decreased in richness, compared to healthy children, with high abundance of Propionibacterium acnes, Staphylococcus spp., and Clostridiaceae-particularly C. difficile, which was only identified in CF patient samples. In conjunction, there was depleted colonization by Blautia, Pseudobutyrivibrio, Roseburia, Faecalibacterium, Anaerostipes, Subdoligranulum, Ruminococcus, Streptococcus, Dorea, and Coprococcus spp. (40). Additional studies identified that infants with CF that have a more diverse gut microbiome profile have delays in time until initial Pseudomonas aeruginosa colonisation and first pulmonary exacerbation event (41), highlighting the gutlung link in this chronic lung disease.

\section{Predicted gut microbiome in COPD}

Currently, there are no published studies of the COPD gut microbiome. Several factors associated with COPD are known to cause dysbiosis of the gut microbiome. Thus, it is predicted that there is dysbiosis of the gut microbiome in
COPD patients. Table 1 summarises the pattern of dysbiosis associated with risk factors (age, gender and tobacco smoking) and clinical factors (use of therapeutic drugs such as oral corticosteroids and antibiotics, BMI and diet) in COPD. Of these disease factors, diet is readily modifiable and can have a positive impact on COPD outcomes $(15,20,23)$. Furthermore, specific nutritional components within diets are known to influence the gut microbiome. For instance, the Western diet, which is high in animal fats and proteins, promotes decreases in bacterial diversity, encouraging dominant colonization by Bacteroides spp. (57). Conversely, the Prudent diet, which is rich in fruits, vegetables, and legumes, increases diversity through the availability of indigestible fibre for utilization by bacteria as a source of energy. When comparing a Western and a Prudent diet, one of the most notable differences is the amount of dietary fibre. The Western diet is notoriously deficient in dietary fibre.

\section{Dietary fibre as a therapeutic agent in COPD- addressing the 'fibre gap'}

Chronic airway inflammation is one of the main drivers of COPD pathogenesis and is often attributed to perpetuated acute inflammation from long-term exposure to noxious inhalants such as biomass smoke or cigarette smoke (58). The understanding of COPD pathogenesis is focused on the pathological changes occurring the airways. However, the impact of these exposures can occur distally to the lungs. For instance, cigarette smoke exposure has been shown to increase systemic inflammation as well as perturb the gut microbiota $(2,59)$. Cigarette smoking is the most significant risk factor for COPD and smoking cessation is the most effective intervention for the prevention and management of COPD (1). However, the condition of many patients continue to deteriorate even with smoking cessation. After smoking cessation, management strategies are focused on respiratory-based interventions. In a new approach, cigarette smoke has been shown to reduce the diversity of the microbes in the gut (59), which is a characteristic of the disease-associated microbiota (10). A diet lacking fermentable fibre can result in malnourishment of the microbiota, which can lead to gut dysbiosis and the promotion of local and systemic chronic inflammation (60). Thus, it is plausible that gut dysbiosis may well be another mediator in the pathogenesis of COPD attributed to cigarette smoking. Consequently, gut-based interventions that repair microbial dysbiosis by addressing dietary fibre 
Table 1 Risk and clinical factors that may contribute to dysbiosis of the gut microbiome in COPD

\begin{tabular}{|c|c|c|}
\hline Risk factor & Characteristics of dysbiosis & Reference \\
\hline \multirow{3}{*}{ Age } & Decrease in species (taxa) within a single person & \\
\hline & Reduction of Bacteroides, Bifidobacteria and Lactobacilli & \\
\hline & Increase in Enterobacteria, C. perfringens and C. difficile & \\
\hline \multirow[t]{3}{*}{ Cigarette smoking } & Decreased alpha diversity and richness & $(45)$ \\
\hline & Decreased Bifidobacteria & \\
\hline & Increased Proteobacteria and Enterobacteria & \\
\hline \multirow[t]{2}{*}{ Use of therapeutic drugs } & Corticosteroids & $(46-49)$ \\
\hline & Decrease in $\alpha$-diversity and abundance & \\
\hline \multirow[t]{4}{*}{ BMI } & Normal weight associated with Bifidobacterium and Lactobacillus & $(50-53)$ \\
\hline & Increased ration of Firmicutes/Bacteroidetes (F/B) in obesity & \\
\hline & Obesity is strongly associated with the Firmicutes & \\
\hline & Healthy weight is strongly associated with the Bacteroidetes phylum & \\
\hline \multirow[t]{2}{*}{ Gender } & Increased abundance of Veillonella and Methanobrevibacter in men versus women & $(54)$ \\
\hline & Decreased abundance of Bilophila in men versus women & \\
\hline Diet & Increase/decrease diversity, richness and abundance of most bacteria & $(55,56)$ \\
\hline
\end{tabular}

COPD, chronic obstructive pulmonary disease; SCFA, short chain fatty acid.

intake may have a strong impact in the prevention and management of COPD.

Dietary fibre intake has a significant impact on the diversity and abundance of microbiota in the gut microbiome, with different fibre sources having varied effects $(61,62)$. Supplementation with fermentable fibres has positive impacts on individuals with chronic disease due to the capacity to promote the growth of a health gut microbiome and increase SCFA production by commensal bacteria (27). Unfortunately, the Western diet is deprived of microbiota-accessible fibre and contributes to reduced microbial diversity. Low fibre diet can promote chronic inflammatory diseases such as inflammatory bowel disease, cancer, allergies, autoimmune disease and obesity and its associated pathologies. Furthermore, a Western diet is high in saturated fat that may promote inflammation $(14,17,63,64)$. There is the potential, at least in part, for these diseases to be prevented or delayed by addressing the 'fibre gap' through adjustment of diet (65). In accordance with this, a high fibre diet has been shown to reduce the risk of COPD. Szmidt et al., (2019) investigated the association between baseline and long-term intake of dietary fibre and COPD risk in a population-based prospective cohort of 35,339 Swedish women (66). They found that longterm high dietary fibre intake was associated with a $30 \%$ lower risk of COPD, which was specific to cereal and fruit fibres. This reduced risk was present in current and former smokers and absent in never smokers. Furthermore, a randomized controlled trial found that a dietary intervention to increase the consumption of fruits and vegetables, known to be high in fibre, was associated with improved lung function (67).

The positive effects observed from high dietary fibre intake and the increase in fibrous foods is thought to be the result of the production of SCFAs from the digestion of fibre by commensal gut bacteria. SCFAs are organic products 
mainly composed of acetate, propionate and butyrate, which possess key roles in regulating host metabolism, immune system, and cell proliferation (34). SCFAs are found at high concentrations in the gut and are readily absorbed into the blood stream where they are transported to the peripheral circulation via the portal vein to act on the liver (68) and peripheral tissues (34). Though the levels of SCFAs are low in the peripheral circulation, they act as signalling molecules and regulate inflammation (34). Recent research in asthma has investigated the acute effect a single dose of dietary fibre in airway inflammation and expression of free fatty acids receptors in asthma. Adult asthmatics received either a soluble fibre meal $(\mathrm{N}=17)$ or a placebo meal $(\mathrm{N}=12)$ of simple carbohydrates. The study showed that a single dose of fermentable fibre effectively reduced airway inflammation, which coincided with increases in blood SCFA and the expression of GRP41 and GPR43 that regulate immune processes (69). Four hours after consumption, sputum total cell count, neutrophils, macrophages, lymphocytes and IL- 8 were significantly reduced compared to the control group (69).

\section{Conclusions}

The metabolism of dietary fibre can provide local and systemic anti-inflammatory effects. Recent research indicates that food and specific nutrients, such as dietary fibre, consumed in a balanced diet, are associated with better lung function and reduced COPD risk. Collectively, research supports the premise that increasing dietary fibre intake may be an effective therapeutic approach to treat chronic inflammation, the underlying cause of COPD pathogenesis. By addressing the 'fibre gap' in the diet of COPD patients, targeted dietary intervention may reduce inflammation through the repair of gut dysbiosis and increased SCFA production. Current management strategies are focused and effective in alleviating patient symptoms and preventing exacerbations of COPD. However, to progress and improve management, a paradigm shift from reactive to predictive, preventative, personalized and participatory medicine is needed (70). The continued improvement of our knowledge regarding the influence of diet on COPD will provide health professionals with evidence-based lifestyle interventions to improve patient outcomes.

\section{Acknowledgments}

Funding: A Vaughan is supported by The Prince Charles
Hospital Foundation Research Fellowship and Innovation grant (RF2017-05 and INN2018-30). IA Yang is supported by a National Health and Medical Research Council of Australia Project grant (APP1121740). PM Hansbro is supported by fellowships from the National Health and Medical Research Council of Australia (1079187, 1175134).

\section{Footnote}

Conflicts of Interest: The authors have no conflicts of interest to declare.

Ethical Statement: The authors are accountable for all aspects of the work in ensuring that questions related to the accuracy or integrity of any part of the work are appropriately investigated and resolved.

\section{References}

1. Yang IA, Brown JL, George J, et al. COPD-X Australian and New Zealand guidelines for the diagnosis and management of chronic obstructive pulmonary disease: 2017 update. Med J Aust 2017;207:436-42.

2. Jones B, Donovan C, Liu G, et al. Animal models of COPD: What do they tell us? Respirology 2017;22:21-32.

3. Vos T, Flaxman AD, Naghavi M, et al. Years lived with disability (YLDs) for 1160 sequelae of 289 diseases and injuries 1990-2010: a systematic analysis for the Global Burden of Disease Study 2010. Lancet 2012;380:2163-96.

4. López-Campos JL, Tan W, Soriano JB. Global burden of COPD. Respirology 2016;21:14-23.

5. Keely S, Talley NJ, Hansbro PM. Pulmonary-intestinal cross-talk in mucosal inflammatory disease. Mucosal Immunol 2012;5:7.

6. Caramori G, Ruggeri P, Mumby S, et al. Molecular links between COPD and lung cancer: new targets for drug discovery? Expert Opin Ther Targets 2019;23:539-53.

7. Ng Kee Kwong F, Nicholson AG, Harrison CL, et al. Is mitochondrial dysfunction a driving mechanism linking COPD to nonsmall cell lung carcinoma? Eur Respir Rev 2017;26:170040.

8. Hopkins RJ, Duan F, Chiles C, et al. Reduced Expiratory Flow Rate among Heavy Smokers Increases Lung Cancer Risk. Results from the National Lung Screening TrialAmerican College of Radiology Imaging Network Cohort. Ann Am Thorac Soc 2017;14:392-402.

9. Simpson JL, Powell H, Baines KJ, et al. The effect of azithromycin in adults with stable neutrophilic COPD: a 
double blind randomised, placebo controlled trial. PloS One 2014;9:e105609.

10. Budden KF, Gellatly SL, Wood DL, et al. Emerging pathogenic links between microbiota and the gut-lung axis. Nat Rev Microbiol 2017;15:55.

11. Schols AM, Ferreira IM, Franssen FM, et al. Nutritional assessment and therapy in COPD: a European Respiratory Society statement. Eur Respir J 2014;44:1504-20.

12. Budden KF, Shukla SD, Rehman SF, et al. Functional effects of the microbiota in chronic respiratory disease. Lancet Respir Med 2019;7:907-20.

13. Shukla SD, Budden KF, Neal R, et al. Microbiome effects on immunity, health and disease in the lung. Clin Transl Immunology 2017;6:e133.

14. Wood LG, Li Q, Scott HA, et al. Saturated fatty acids, obesity, and the nucleotide oligomerization domain-like receptor protein 3 (NLRP3) inflammasome in asthmatic patients. J Allergy Clin Immunol 2019;143:305-15.

15. Kimokoti RW, Millen BE. Nutrition for the Prevention of Chronic Diseases. Med Clin North Am 2016;100:1185-98.

16. Chambers DC, Gellatly SL, Hugenholtz P, et al. JTD special edition 'Hot Topics in COPD'-The microbiome in COPD. J Thorac Dis 2014;6:1525.

17. Rutting S, Zakarya R, Bozier J, et al. Dietary fatty acids amplify inflammatory responses to infection through p38 MAPK signaling. Am J Respir Cell Mol Biol 2019;60:554-68.

18. Martinez KB, Leone V, Chang EB. Western diets, gut dysbiosis, and metabolic diseases: Are they linked? Gut Microbes 2017;8:130-42.

19. Kaidar-Person O, Person B, Szomstein S, et al. Nutritional Deficiencies in Morbidly Obese Patients: A New Form of Malnutrition? Obes Surg 2008;18:870-6.

20. Shaheen SO, Jameson KA, Syddall HE, et al. The relationship of dietary patterns with adult lung function and COPD. Eur Respir J 2010;36:277-84.

21. Mekary RA. A higher overall diet quality is inversely associated with the risk of chronic obstructive pulmonary disease (COPD) in men and women. Evid Based Med 2016;21:36.

22. Varraso R, Chiuve SE, Fung TT, et al. Alternate Healthy Eating Index 2010 and risk of chronic obstructive pulmonary disease among US women and men: prospective study. BMJ 2015;350:h286.

23. Young RP, Hopkins RJ. Is the "Western diet" a new smoking gun for chronic obstructive pulmonary disease? Ann Am Thorac Soc 2018;15:662-3.

24. Kaluza J, Harris H, Wallin A, et al. Dietary Fiber Intake and Risk of Chronic Obstructive Pulmonary Disease:
A Prospective Cohort Study of Men. Epidemiology 2018;29:254-60.

25. Kaluza J, Harris HR, Linden A, et al. Long-term consumption of fruits and vegetables and risk of chronic obstructive pulmonary disease: a prospective cohort study of women. Int J Epidemiol 2018;47:1897-909.

26. Kaluza J, Larsson SC, Orsini N, et al. Fruit and vegetable consumption and risk of COPD: a prospective cohort study of men. Thorax 2017;72:500-9.

27. Wang B, Yao M, Lv L, et al. The Human Microbiota in Health and Disease. Engineering 2017;3:71-82.

28. Keely S, Hansbro PM. Lung-gut cross talk: a potential mechanism for intestinal dysfunction in patients with COPD. Chest 2014;145:199-200.

29. Fricker M, Goggins BJ, Mateer S, et al. Chronic cigarette smoke exposure induces systemic hypoxia that drives intestinal dysfunction. JCI Insight 2018;3. doi: 10.1172/jci. insight. 94040.

30. Mateer SW, Maltby S, Marks E, et al. Potential mechanisms regulating pulmonary pathology in inflammatory bowel disease. J Leukoc Biol 2015;98:727-37.

31. Mateer SW, Mathe A, Bruce J, et al. IL-6 drives neutrophil-mediated pulmonary inflammation associated with bacteremia in murine models of colitis. Am J Pathol 2018;188:1625-39.

32. Liu G, Mateer SW, Hsu A, et al. Platelet activating factor receptor regulates colitis-induced pulmonary inflammation through the NLRP3 inflammasome. Mucosal Immunol 2019:12:862-73.

33. Anand S, Mande SS. Diet, Microbiota and Gut-Lung Connection. Front Microbiol 2018;9:2147.

34. Ratajczak W, Ryl A, Mizerski A, et al. Immunomodulatory potential of gut microbiome-derived short-chain fatty acids (SCFAs). Acta Biochim Pol 2019;66:1-12.

35. Marsland BJ, Trompette A, Gollwitzer ES. The Gut-Lung Axis in Respiratory Disease. Ann Am Thorac Soc 2015;12 Suppl 2:S150-6.

36. Lloyd-Price J, Abu-Ali G, Huttenhower C. The healthy human microbiome. Genome Med 2016;8:51.

37. Arumugam M, Raes J, Pelletier E, et al. Enterotypes of the human gut microbiome. Nature 2011;473:174.

38. Wang Q, Li F, Liang B, et al. A metagenome-wide association study of gut microbiota in asthma in UK adults. BMC Microbiol 2018;18:114.

39. Furusawa Y, Obata Y, Fukuda S, et al. Commensal microbe-derived butyrate induces the differentiation of colonic regulatory T cells. Nature 2013;504:446.

40. Vernocchi P, Del Chierico F, Russo A, et al. Gut 
microbiota signatures in cystic fibrosis: Loss of host CFTR function drives the microbiota enterophenotype. PloS One 2018;13:e0208171.

41. Hoen AG, Li J, Moulton LA, et al. Associations between gut microbial colonization in early life and respiratory outcomes in cystic fibrosis. J Pediatr 2015;167:138-47.e1.

42. Nagpal R, Mainali R, Ahmadi S, et al. Gut microbiome and aging: Physiological and mechanistic insights. Nutr Healthy Aging 2018;4:267-85.

43. Odamaki T, Kato K, Sugahara H, et al. Age-related changes in gut microbiota composition from newborn to centenarian: a cross-sectional study. BMC Microbiol 2016;16:90.

44. O'Toole PW, Jeffery IB. Gut microbiota and aging. Science 2015;350:1214-5.

45. Savin Z, Kivity S, Yonath H, et al. Smoking and the intestinal microbiome. Arch Microbiol 2018;200:677-84.

46. Huang EY, Inoue T, Leone VA, et al. Using Corticosteroids to Reshape the Gut Microbiome: Implications for Inflammatory Bowel Diseases. Inflamm Bowel Dis 2015;21:963-72.

47. Yoon MY, Yoon SS. Disruption of the Gut Ecosystem by Antibiotics. Yonsei Med J 2018;59:4-12.

48. Panda S, Casellas F, Vivancos JL, et al. Short-term effect of antibiotics on human gut microbiota. PloS One 2014;9:e95476.

49. Ferrer M, Méndez-García C, Rojo D, et al. Antibiotic use and microbiome function. Biochem Pharmacol 2017;134:114-26.

50. Castaner O, Goday A, Park YM, et al. The gut microbiome profile in obesity: a systematic review. Int J Endocrinol 2018;2018:4095789.

51. Kasai C, Sugimoto K, Moritani I, et al. Comparison of the gut microbiota composition between obese and nonobese individuals in a Japanese population, as analyzed by terminal restriction fragment length polymorphism and next-generation sequencing. BMC Gastroenterol 2015;15:100.

52. Million M, Maraninchi M, Henry M, et al. Obesityassociated gut microbiota is enriched in Lactobacillus reuteri and depleted in Bifidobacterium animalis and Methanobrevibacter smithii. Int J Obes (Lond) 2012;36:817.

53. Hu H-J, Park S-G, Jang HB, et al. Obesity alters the microbial community profile in Korean adolescents. PLoS One 2015;10:e0134333.

54. Haro C, Rangel-Zúñiga OA, Alcalá-Díaz JF, et al. Intestinal microbiota is influenced by gender and body mass index. PloS One 2016;11:e0154090.

55. David LA, Maurice CF, Carmody RN, et al. Diet rapidly and reproducibly alters the human gut microbiome. Nature 2014;505:559.

56. Turnbaugh PJ, Ridaura VK, Faith JJ, et al. The Effect of Diet on the Human Gut Microbiome: A Metagenomic Analysis in Humanized Gnotobiotic Mice. Sci Transl Med 2009; 1:6ra14.

57. Senghor B, Sokhna C, Ruimy R, et al. Gut microbiota diversity according to dietary habits and geographical provenance. Human Microbi J 2018;7-8:1-9.

58. Shaw JG, Vaughan A, Dent AG, et al. Biomarkers of progression of chronic obstructive pulmonary disease (COPD). J Thorac Dis 2014;6:1532-47.

59. Sze MA, Hogg JC, Sin DD. Bacterial microbiome of lungs in COPD. Int J Chron Obstruct Pulmon Dis 2014;9:229-38.

60. Chassaing B, Vijay-Kumar M, Gewirtz AT. How diet can impact gut microbiota to promote or endanger health. Curr Opin Gastroenterol 2017;33:417-21.

61. Simpson HL, Campbell BJ. Review article: dietary fibre-microbiota interactions. Aliment Pharmacol Ther 2015;42:158-79.

62. Pylkas AM, Juneja LR, Slavin JL. Comparison of Different Fibers for In Vitro Production of Short Chain Fatty Acids by Intestinal Microflora. J Med Food 2005;8:113-6.

63. Periyalil HA, Wood L, Wright T, et al. Obese asthmatics are characterized by altered adipose tissue macrophage activation. Clin Exp Allergy 2018;48:641-9.

64. Rutting S, Xenaki D, Lau E, et al. Dietary omega-6, but not omega-3, polyunsaturated or saturated fatty acids increase inflammation in primary lung mesenchymal cells. Am J Physiol Lung Cell Mol Physiol 2018;314:L922-35.

65. Makki K, Deehan EC, Walter J, et al. The Impact of Dietary Fiber on Gut Microbiota in Host Health and Disease. Cell Host Microbe 2018;23:705-15.

66. Szmidt MK, Kaluza J, Harris HR, et al. Long-term dietary fiber intake and risk of chronic obstructive pulmonary disease: a prospective cohort study of women. Eur J Nutr 2019. [Epub ahead of print].

67. Keranis E, Makris D, Rodopoulou P, et al. Impact of dietary shift to higher-antioxidant foods in COPD: a randomised trial. Eur Respir J 2010;36:774-80.

68. Young RP, Hopkins RJ, Marsland B. The gut-liver-lung axis. Modulation of the innate immune response and its possible role in chronic obstructive pulmonary disease. Am J Respir Cell Mol Biol 2016;54:161-9.

69. Halnes I, Baines KJ, Berthon BS, et al. Soluble Fibre Meal Challenge Reduces Airway Inflammation and Expression 
of GPR43 and GPR41 in Asthma. Nutrients 2017;9:57.

70. Schols AMWJ. The 2014 ESPEN Arvid Wretlind Lecture:
Metabolism \& nutrition: Shifting paradigms in COPD management. Clin Nutr 2015;34:1074-9.

Cite this article as: Vaughan A, Frazer ZA, Hansbro PM, Yang IA. COPD and the gut-lung axis: the therapeutic potential of fibre. J Thorac Dis 2019;11(Suppl 17):S2173-S2180. doi: 10.21037/ jtd.2019.10.40 